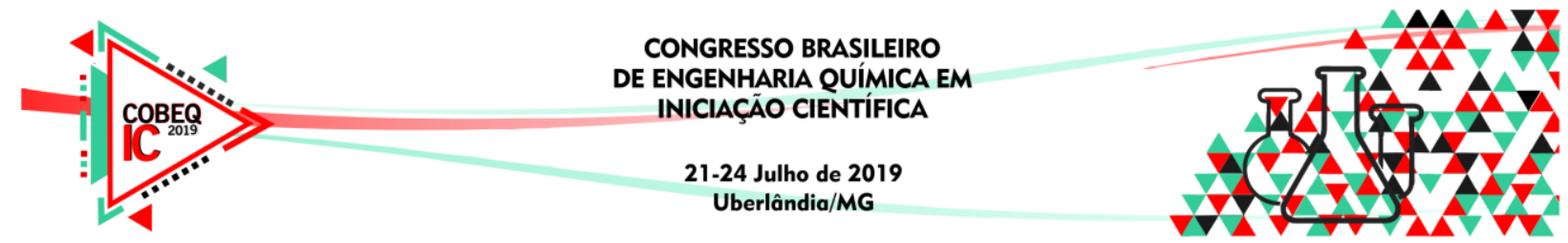

\title{
SIMULAÇÃO DO COMPORTAMENTO HIDRODINÂMICO DE UM LEITO DE JORRO: COMPARAÇÃO ENTRE MÉTODO DOS ELEMENTOS DISCRETOS (DEM) E MODELO DE DOIS FLUIDOS (TFM)
}

\author{
L. P. BORGES ${ }^{1}$, R. BÉTTEGA ${ }^{1}$ \\ ${ }^{1}$ Universidade Federal de São Carlos, Departamento de Engenharia Química \\ E-mail para contato: borgeslaira@ hotmail.com
}

\begin{abstract}
RESUMO - Leitos de jorro são equipamentos que favorecem os fenômenos de transferência de calor e massa uma vez que, as partículas são movimentadas de forma cíclica em seu interior. O conhecimento da fluidodinâmica do equipamento é importante na compreensão desses fenômenos de transferência, além de auxiliar nos projetos de construção e aplicações do mesmo. A aplicação da fluidodinâmica computacional (CFD) no estudo de leitos de jorro tem se intensificado com os avanços tecnológicos de software e hardware. Existem basicamente duas abordagens para modelagem do escoamento gás-sólido no equipamento: abordagem Euleriana-Euleriana, que utiliza o modelo CFD-TFM; e a abordagem Euleriana-Lagrangeana, que utiliza o acoplamento CFD-DEM. A velocidade de mínimo jorro é um parâmetro importante do equipamento que pode ser facilmente obtido experimentalmente e possibilita a avaliação dos resultados simulados numericamente, indicando a qualidade da modelagem e do procedimento numérico adotado no simulador. Neste contexto, o objetivo deste trabalho foi a simulação numérica da fluidodinâmica de um leito de jorro encontrado na literatura utilizando as técnicas de CFD-DEM e CFD-TFM e comparação dos resultados obtidos por ambas as técnicas.
\end{abstract}

\section{INTRODUÇÃO}

O leito de jorro, proposto por Mathur e Gishler (1955), era aplicado inicialmente em processos de secagem, granulação e recobrimento ((Mathur e Epstein, 1974). Com o desenvolvimento tecnológico, passou a ser empregado também em processos de pirólise de biomassa, reações de polimerização e gaseificação de biomassa e carvão.

O equipamento consiste em um vaso aberto no topo com base cônica. É caracterizado por três regiões: canal de jorro, fonte e ânulo. O fluido é injetado verticalmente por um orifício na região central da base do vaso. Com velocidade alta o suficiente, o fluido faz com que um fluxo de partículas suba na região central do leito, formando um canal oco, chamado de canal de jorro. As partículas são transportadas até uma altura superior à do leito, formando a região de fonte. Quando a componente da velocidade paralela à subida é zerada, o movimento das partículas é invertido e elas retornam para o leito, na região anular, localizada entre o canal e a parede do equipamento (Epstein e Grace, 2011). 


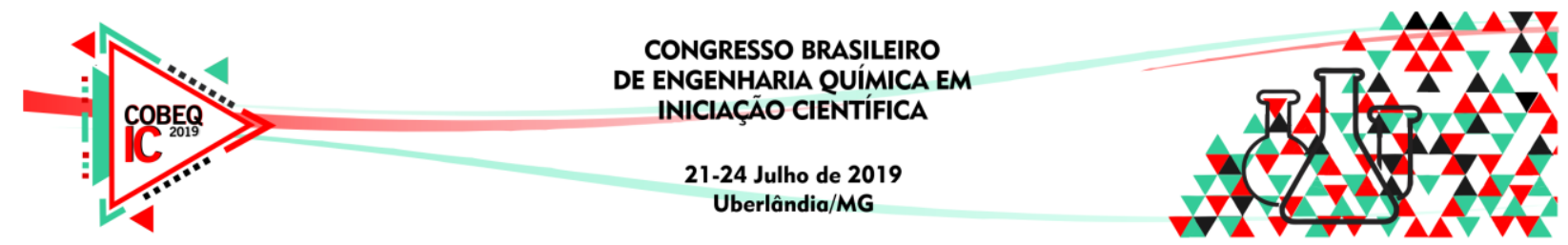

Existem basicamente duas abordagens para modelagem de leitos de jorro: Euler-Euler e Euler-Lagrange. Na abordagem Euler-Euler, fluido e partículas são tratados como contínuos interpenetrantes, e a modelagem de ambas as fases presentes no escoamento é realizada por meio das equações de conservação de quantidade de movimento, sendo a teoria cinética granular aplicada nas equações que representam a fase particulada como um pseudo-fluido, e associa-se à essa abordagem o Modelo dos Dois Fluidos (TFM - Two Fluid Model). Quanto à abordagem Euler-Lagrange, o fluido continua sendo tratado como contínuo, enquanto o movimento da fase sólida é representado pela $2^{\text {a }}$ Lei de Newton, e cada partícula é tratada individualmente. O Método dos Elementos Discretos (DEM - Discrete Element Method) pode ser utilizado na abordagem Euler-Lagrange para o estudo de leitos de jorro, e considera as interações partícula-partícula e partícula-parede em seu equacionamento.

Assim, o trabalho tem como objetivo principal a comparação entre simulações utilizando as modelagens CFD-TFM e CFD-DEM, a fim de identificar semelhanças e diferenças nos resultados obtidos por cada metodologia.

\section{METODOLOGIA}

\subsection{Modelagem matemática}

Modelo dos Dois Fluidos: O TFM baseia-se na abordagem Euleriana-Euleriana, onde as fases sólida e fluida são tratadas como contínuos interpenetrantes. A equação da continuidade para cada fase q ( $\mathrm{f}$ - fluido ou $\mathrm{s}$ - sólida), assumindo que não há transferência de massa entre as fases, é expressa pela equação 1 . A equação de quantidade de movimento de cada fase fluida é expressa pela equação 2 e da fase sólida, pela equação 3 .

$$
\begin{aligned}
& \frac{\partial}{\partial t}\left(a_{q} \rho_{q}\right)+\nabla \cdot\left(a_{q} \rho_{q} \vec{u}_{q}\right)=0 \\
& \frac{\partial}{\partial t}\left(a_{f} \rho_{f} \vec{u}_{f}\right)+\nabla \cdot\left(a_{f} \rho_{f} \vec{u}_{f} \vec{u}_{f}\right)=-a_{f} \nabla p+\nabla \cdot \overline{\bar{t}}_{f}+a_{f} \rho_{f} \vec{g}+K_{f s}\left(\vec{u}_{f}-\vec{u}_{s}\right) \\
& \frac{\partial}{\partial t}\left(a_{s} \rho_{s} \vec{u}_{s}\right)+\nabla \cdot\left(a_{s} \rho_{s} \vec{u}_{s} \vec{u}_{s}\right)=-a_{s} \nabla p-\nabla p_{s}+\nabla \cdot \overline{\bar{t}}_{s}+a_{s} \rho_{s} \vec{g}-K_{f s}\left(\vec{u}_{f}-\vec{u}_{s}\right)
\end{aligned}
$$

Os termos $\rho_{q}$ e $\vec{u}_{q}$ são respectivamente densidade e velocidade de cada fase $\mathrm{q}$ e $a_{s}=1-a_{f}, p$ é a pressão compartilhada pelas fases; $p_{s}$ é a pressão dos sólidos; $\overline{\bar{\tau}}_{f}$ é o tensor das tensões; $K_{f s}$ é o coeficiente de troca de quantidade de movimento entre as fases e $\vec{g}$ é a aceleração da gravidade.

Utilizou-se o modelo de Gidaspow et al. (1992) para o cálculo da força de arraste e modelo $k-\varepsilon$ para equacionamento da turbulência.

Acoplamento CFD-DEM: O acoplamento enquadra-se na abordagem Euler-Lagrange onde cada fase apresenta um equacionamento característico. A modelagem da fase fluida continua sendo por meio das equações de conservação de massa e balanço de quantidade de 


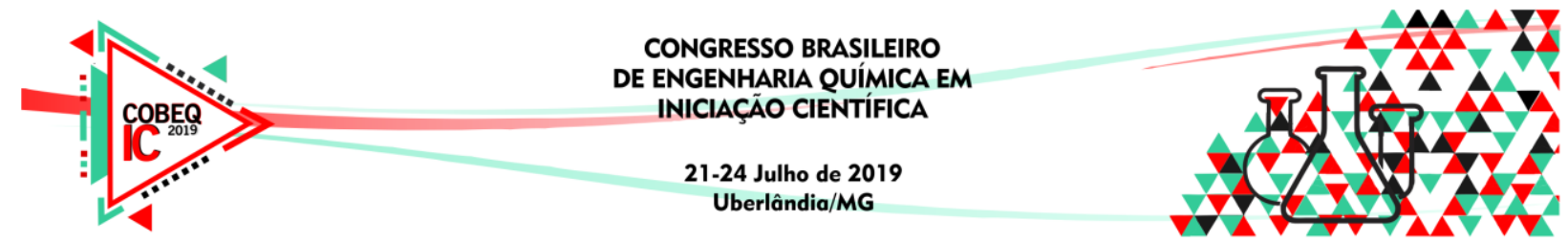

movimento, escritas, respectivamente, pelas equações 4 e 5 . O movimento das partículas da fase sólida é previsto pela equação 6 .

$$
\begin{aligned}
& \frac{\partial}{\partial t}\left(a_{f} \rho_{f}\right)+\nabla \cdot\left(a_{f} \rho_{f} \vec{u}_{f}\right)=0 \\
& \frac{\partial}{\partial t}\left(a_{f} \rho_{f} \vec{u}_{f}\right)+\nabla \cdot\left(a_{f} \rho_{f} \vec{u}_{f} \vec{u}_{f}\right)=-a_{f} \nabla p+\nabla \cdot\left(\mu_{f} \nabla \vec{u}_{f}+\overrightarrow{\vec{t}}_{R_{f}}\right)+a_{f} \rho_{f} \vec{g}+\vec{F} \\
& \frac{d \vec{u}_{p}}{d t}=\frac{\vec{u}-\vec{u}_{p}}{\tau_{r}}+\vec{g} \frac{\rho_{p}-\rho}{\rho_{p}}+\vec{F}_{\text {adicional }}
\end{aligned}
$$

Na equação do balanço de quantidade de movimento, o termo $\vec{F}$ refere-se à todas as forças adicionais por unidade de volume, incluindo as forças de interação com a fase sólida. $\mathrm{O}$ termo $\vec{F}_{\text {adicional }}$ refere-se à aceleração resultante das forças adicionais, como as forças resultantes das colisões das partículas com as paredes e $\tau_{r}$ é o tempo de relaxação da partícula.

O modelo de arraste utilizado foi o de partículas não esféricas, considerando esfericidade 1 das partículas, e modelo $k-\varepsilon$ para a turbulência.

\subsection{Procedimento de solução numérica}

A unidade experimental simulada é apresentada em Marchelli et al. (2017). Devido ao elevado custo computacional requerido pelo acoplamento CFD-DEM, optou-se por utilizar uma geometria pseudo 2D, que consiste em uma seção do leito tridimensional, com uma das dimensões muito menor que as outras, e condição de simetria na direção axial, no centro do leito. A geometria pseudo 2D aplicada à leitos de jorro tem sido utilizada por autores como Mahmoodi et al. (2018) e Moliner et al. (2018).

Na Figura 1 é apresentado o domínio computacional, construído a partir do pacote ANSYS 19.1. A geometria foi gerada utilizando o software Design Modeler e a malha, o software Meshing. A malha é composta majoritariamente por células hexaédricas totalizando 1731 célula e 2584 nós, e foi escolhida após aplicação do teste de independência de malhas GCI (Grid Convergence Index).

Como fase particulada considerou-se esferas de vidro com diâmetro de $1,5 \mathrm{~mm}$ e densidade de $2508 \mathrm{~kg} / \mathrm{m}^{3}$, totalizando 80928 partículas. As simulações consistiram em bateladas de 6 segundos, com 3 segundos iniciais conduzidos em regime laminar e os demais 3 segundos em regime turbulento, com velocidade da fase fluida na entrada prescrita de $21,778 \mathrm{~m} / \mathrm{s}$ e gradiente de velocidade nulo.

Nas simulações o algoritmo Phase Coupled SIMPLE foi utilizado para o acoplamento pressão-velocidade. Na discretização (CFD-TFM), para o cálculo dos gradientes foi utilizado Least Squares Cell Based e para os demais campos foi utilizado First Order Upwind. Os 

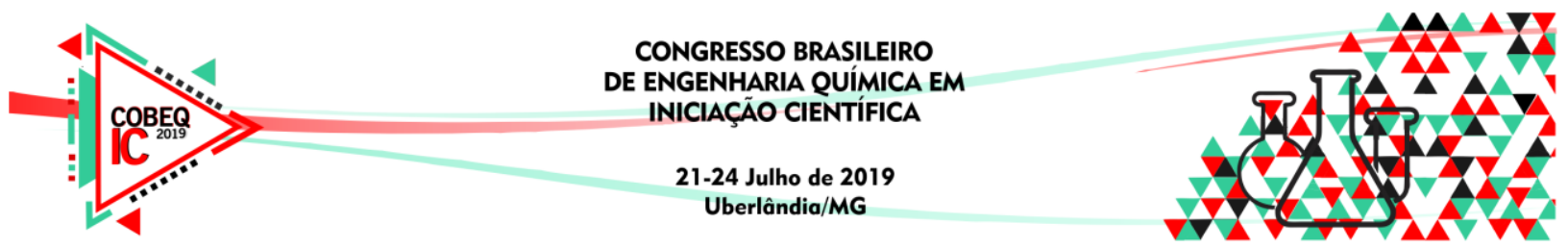

parâmetros de relaxação foram de 0,7 para o momentum, 0,2 para temperatura granular e 0,5 para fração volumétrica.

Quanto ao CFD-DEM, a discretização foi feita utilizando Green-Gauss Node Based para cálculo dos gradientes e QUICK para o momentum e fração volumétrica. Os parâmetros de relaxação para as equações de momentum e fase discreta foram, respectivamente, 0,1 e 0,5, sendo os demais mantidos conforme o default do Fluent. O critério de convergência para todas as variáveis foi de $1,0 \times 10^{-3}$. Na Tabela 1 são apresentados os demais parâmetros e equações constitutivas utilizadas nas simulações DEM e TFM.

Figura 1 - Domínio computacional.

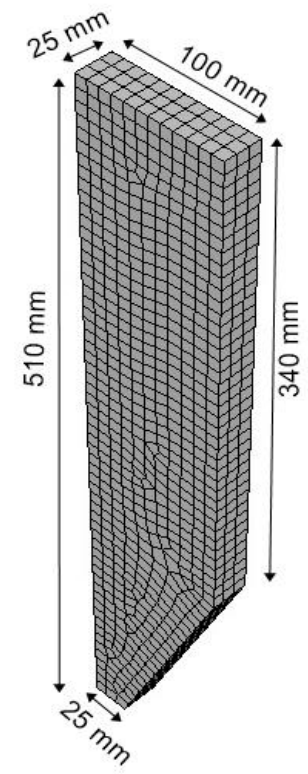

Fonte: Elaborado pela autora.

Tabela 1 - Parâmetros e equações constitutivas das simulações TFM e DEM.

\begin{tabular}{|c|c|c|c|}
\hline \multicolumn{2}{|c|}{ CFD-TFM } & \multicolumn{2}{c|}{ CFD-DEM } \\
\hline Viscosidade granular & Gidaspow & Lei da força normal de contato & Spring-dashpot \\
\hline Viscosidade granular bulk & Lun et al. & Lei da força tangencial de contato & Friction-dshf-rolling \\
\hline Viscosidade de fricção & Schaeffer & $\mathrm{K}$ - modelo Spring-Dashpot & $100 \mathrm{~N} / \mathrm{m}$ \\
\hline Angulo de fricção & 30 & $\eta$ - modelo Spring-Dashpot & 0,5 \\
\hline Distribuição radial & Lun et al. & $\mu_{\text {stick }}$ & 0,5 \\
\hline Modulo de elasticidade & Derived & $\mu_{\text {glide }}$ & 0,2 \\
\hline Pressão de fricção & Based kgtf & $\mu_{\text {limit }}$ & 0,1 \\
\hline Modulo de fricção & Derived & $\mathrm{V}_{\text {glide }}$ & $1 \mathrm{~m} / \mathrm{s}$ \\
\hline Limite de atrito de fricção & 0,62 & $\mathrm{~V}_{\text {limit }}$ & $10 \mathrm{~m} / \mathrm{s}$ \\
\hline Temperatura granular & Algebraic & Slope limit & $100 \mathrm{~s}$ \\
\hline Pressão dos sólidos & Lun et al. & Time step fluido & $2,0 \times 10^{-5}$ \\
\hline Limite de empacotamento & 0,62 & Time step solido & 200 \\
\hline Time step fluido & $1,0 \times 10^{-4}$ & Máximo de iterações & \\
\hline Máximo de iterações & 100 & & \\
\hline
\end{tabular}




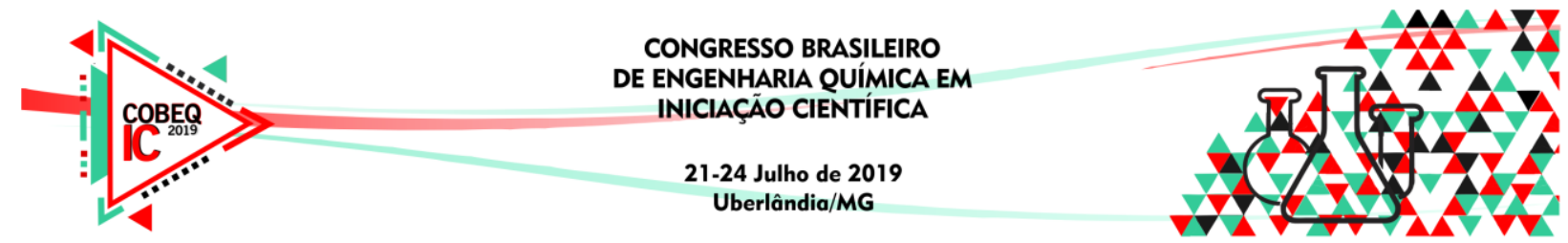

\section{RESULTADOS E DISCUSSÃO}

Na Figura 2 são apresentados os contorno de fração volumétrica de sólidos para as abordagens TFM e DEM. Nota-se nas duas imagens a formação do jorro estável, com todas as regiões características definidas.

Para o acoplamento CFD-DEM, observa-se uma região de fonte definida e preenchida. O canal de jorro também se encontra preenchido com partículas, com uma maior concentração em sua base. O diâmetro do canal é maior na entrada de ar e tende a diminuir à medida que se aproxima da fonte.

Para a modelagem TFM, a região de entrada apresenta baixa concentração de sólidos e o canal de jorro apresenta um diâmetro maior que a entrada de ar, indicando uma suspensão da fase sólida. Observa-se também a variação do diâmetro do canal de jorro, refletindo em pequenos pulsos na fonte, fenômeno observado experimentalmente.

As alturas de fontes e diâmetro de canal de jorro são diferentes em cada abordagem. Com o DEM a altura da fonte chega em $33 \mathrm{~cm}$ enquanto que para o TFM, atinge apenas 15 $\mathrm{cm}$. Quanto ao canal, o diâmetro com o DEM é de cerca de $1,5 \mathrm{~cm}$, enquanto para o TFM esse diâmetro chega à $3 \mathrm{~cm}$. Tais diferenças podem ser associadas aos modelos adotados, visto que os equacionamentos apresentam parâmetros comuns e exclusivos. Equacionamentos diferentes com parâmetros diferentes levam à resultados distintos. Moliner et al. (2019, no prelo) também verificaram que a altura de leito obtida via CFD-DEM era maior que a obtida por CFD-TFM, com o resultado do modelo TFM mais próximo ao experimental.

Figura 2 - Fração de sólidos para as abordagens CFD-TFM e CFD-DEM.

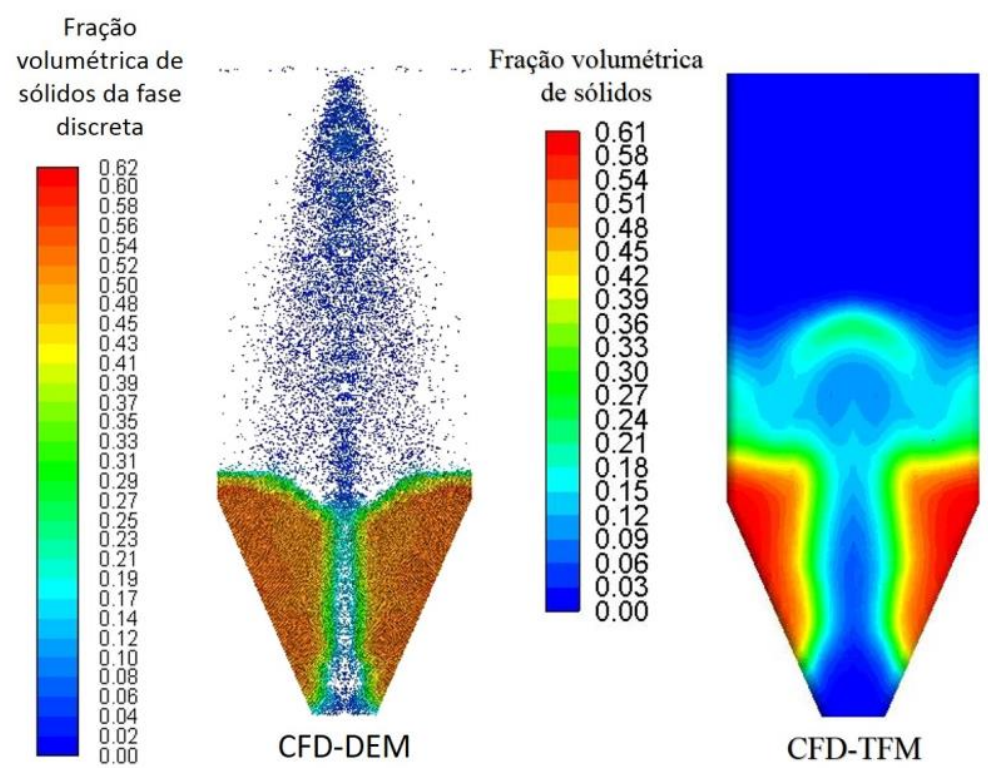

Fonte: Elaborado pela autora. 


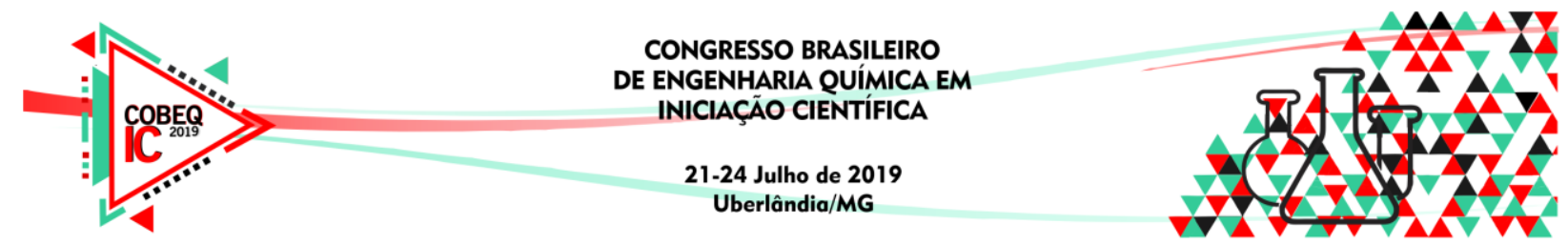

O tempo de simulação também é uma variável diferente para a duas modelagens. A simulação com o DEM demorou cerca de 20 dias, já a simulação com TFM, levou 2 dias. Embora o CFD-DEM resulte em mais informações a respeito das partículas, o conjunto de equações a serem resolvidas é maior, resultando em um custo computacional elevado. Para situações com um número de pequeno de partículas a aplicação do DEM é viável, porém para a escala industrial o tempo para obtenção dos resultados tende a ser grande.

\section{CONCLUSÕES}

As abordagens CFD-TFM e CFD-DEM são capazes de reproduzir o fenômeno do jorro de um leito, considerando a presença das três regiões características do equipamento. Embora a abordagem com CFD-DEM apresente resultados visuais semelhantes ao experimental, a abordagem CFD-TFM requer um menor custo computacional, mesmo para sistemas complexos. Assim, a escolha da modelagem requer ponderação entre o grau de precisão dos dados e o custo computacional.

\section{REFERÊNCIAS}

EPSTEIN, N; GRACE, J. R. Spouted and Spout-Fluid Beds: Fundamentals and Applicantions. New York-USA: Cambridge University Press, 2011.

GIDASPOW, D.; BEZBURUAH, R.; D, J. Hydrodynamics of circulating fluidized beds, Kinetic Theory Approach, Fluidization VII. 1992, [S.1.]: Proceedings of the 7th Engineering Foudation Conference on Fluidization, 1992. p. 75-82.

MAHMOODI, B.; HOSSEINI, S. H.; AHMADI, G. CFD-DEM simulation of a pseudo-twodimensional spouted bed comprising coarse particles. Particuology, 2018.

MARCHELLI, F.; BOVE, D.; MOLINER, C.; BOSIO, B.; ARATO, E. Discrete element method for the prediction of the onset velocity in a spouted bed. Powder Technology, v. 321, p. 119-131, 2017.

MATHUR, K. B.; EPSTEIN, Normam. Spouted Beds. New York-USA: Academic Press, 1974.

MATHUR, K. B.; GISHLER, P. E. A Technique for Contacting Gases with Coarse Solid Particles. American Institute of Chemical Engineers Journal, v. 1, p. 157-164, 1955.

MOLINER, C.; MARCHELLI, F.; SPANACHI, N.; MARTINEZ-FELIPE, A.; BOSIO, B.; ARATO, E. CFD simulation of a Spouted Bed: comparison between the Discrete Element Method (DEM) and the Two Fluid Method (TFM). Chemical Engineering Journal. No prelo 2019. 